\title{
GOLDENER, GRÜNER UND „ANDERER“ WEG ZU OPEN AC- CESS: STATISTIKEN FÜR ÖSTERREICH
}

\author{
von Nora Schmidt
}

\section{Inhalt}

1. Fragestellungen

2. Methoden der Datenerhebung und -bearbeitung

3. Zentrale Ergebnisse der Studie von Science-Metrix

4. Hervorzuhebende Ergebnisse für Österreich

5. Fazit

Zusammenfassung: Archambault et al. veröffentlichten 2014 einen Report, der die Open-Access-(OA)-Raten für Publikationen der Jahre 2008-2013 aus allen Ländern des Europäischen Forschungsraums (ERA) sowie für Brasilien, Kanada, Japan und die USA enthält. Unterschieden wird dabei nicht nur nach Disziplinen, sondern auch nach drei OA-Kategorien: Gold, Grün und einer Restkategorie. Einige wichtige Ergebnisse der Studie werden zusammengefasst und die Daten für Österreich mit jenen des untersuchten Gesamtraums verglichen. Im Ergebnis zeigt sich, dass Österreich in fast allen Disziplinen überdurchschnittlich hohe OA-Raten aufweist. Der Fachbereich „Philosophy \& Theology" bildet dabei jedoch eine Ausnahme. Außerdem scheint das Zugänglichmachen über Soziale Netzwerke und persönliche Homepages in fast allen Fachbereichen beliebter zu sein als der Goldene oder der Grüne Weg. Das gilt insbesondere für die Geistes- und Sozialwissenschaften. Die Daten der Study of Open Access Publishing (SOAP) lassen darauf schließen, dass es OA-Journals in diesen Disziplinen noch an Reputation mangelt.

Schlüsselwörter: Open Access in Österreich; Goldener Weg des Open Access; Grüner Weg des Open Access; Akademische Soziale Netzwerke

\section{GOLDEN, GREEN AND „OTHER“ ROAD TO OPEN ACCESS: STA- TISTICS FOR AUSTRIA}

Abstract: In 2014, Archambault et al. published a report which provided data about Open Access publishing for the years 2008-2013 for all countries of the European Research Area (ERA) as well as for Brazil, Canada, Japan and the USA. They differentiated not only by disciplines but also by three OA categories: Golden, Green and a residual category. Some important results of the study are summarized here. The data for Austria is compared to those from the whole area examined. The results show 
above-average rates for Austria in almost all disciplines. However, the field „Philosophy \& Theology" is an exception. Furthermore, it seems to be more popular in almost all fields to make articles available in Social Networks or on personal homepages than the Golden or the Green road are. That is especially true for the humanities and social sciences. Data from the Study of Open Access Publishing (SOAP) suggest that OA journals are lacking reputation in this fields.

Keywords: Open Access in Austria, Golden Road to Open Access, Green Road Open Access, Academic Social Networks

\section{Fragestellungen}

Wie weit ist Open Access (OA) in Österreich bereits verbreitet - im Vergleich mit anderen Ländern? In welchen Disziplinen wird am ehesten OA publiziert? Welche Bedeutung hat dabei der Grüne Weg des OA, also das Archivieren von Artikeln auf Dokumentenservern? Wie viele Artikel sind weder „Goldene“ OA-Publikationen, die unmittelbar in vollständig frei zugänglichen Zeitschriften erscheinen, noch „Grüne“, sondern werden über Soziale Netzwerke oder persönliche Webseiten zugänglich gemacht? Bislang lagen keine Daten vor, mit deren Hilfe sich diese Fragen beantworten ließen. ${ }^{1}$ Abhilfe schafft nun ein Forschungsprojekt, das durch die Europäische Kommission in Auftrag gegeben und von Science-Metrix durchgeführt wurde (Archambault et al. 2014)2 .

Zunächst werde ich einerseits in die Methoden der Datenerhebung und in die wichtigsten Ergebnisse von Science-Metrix einführen und andererseits meine Methode der Datenextraktion und -auswertung erläutern. Schließlich gilt es, hervorstechende Ergebnisse des Vergleichs der OA-Raten von österreichischen Publikationen mit jenen des gesamten untersuchten Raums darzustellen und mit Hilfe der Daten für Österreich aus der Study of Open Access Publishing (SOAP) Schlussfolgerungen zu ziehen.

\section{Methoden der Datenerhebung und -bearbeitung}

Die Definition von OA, die der Datenerhebung von Science-Metrix zugrunde liegt, ist sehr weit: Lediglich die freie Zugänglichkeit zählt, also die Möglichkeit, den Zeitschriftenartikel - andere Publikationstypen sind ausgespart - lesen zu können, und zwar ohne vorher zu einem Login oder einer Zahlung aufgefordert zu werden. Nach der sonst häufig zitierten Definition 
der „Berliner Erklärung über den offenen Zugang zu wissenschaftlichem Wissen“3 von 2003 entspräche also nur ein kleinerer Anteil davon „echtem OA“, zu dem eine frei Lizenz gehört, durch die das jeweilige Werk auch frei weiterverbreitet und genutzt werden kann. So war es mir z. B. nur aufgrund der CC-BY-Lizenz des Reports von Science-Metrix ohne Nachfrage bei den Autorlnnen möglich, die benötigten Daten aus dem PDF zu extrahieren und weiter zu verarbeiten.

Für die Analyse von Science-Metrix wurden drei Varianten von OA unterschieden (5): Grün, Gold und „Other OA“. Grünes OA bezieht sich hier ausschließlich auf Repositorien, die in OpenDOAR ${ }^{4}$ oder ROAR ${ }^{5}$ verzeichnet sind. Die Autorlnnen haben nicht geprüft, ob alle in der Kategorie „Grün“ gezählten Artikel rechtlich überhaupt auf diese Weise zugänglich sein dürften. So ist anzunehmen, dass sich ein beträchtlicher Anteil „Robin Hood OA“ darunter findet: Artikel, die entgegen nutzungsrechtlicher Bestimmungen zugänglich gemacht wurden. PubMed Central ${ }^{6}$, das gemeinhin durchaus als Repositorium geführt wird, ist „Other OA“ zugeordnet, da es die automatisierte Abfrage rechtlich ausschließt.

Während Gold OA alle Artikel aus wenigstens bereits ein Jahr lang in DOAJ verzeichneten Journals meint, ist die Restkategorie „Other OA“ erklärungsbedürftiger: Hierunter zählen alle Beiträge, die in Zeitschriften oder Repositorien zu finden sind, jedoch artikelweise erst nach einer Embargozeit oder unter bestimmten Bedingungen (z. B. der Zahlung einer Gebühr wie beim „Hybriden OA“) freigeschaltet wurden. Des Weiteren kommen Artikel in diese Restkategorie, die über Soziale Netzwerke wie ResearchGate oder CiteSeerX zugänglich sind. Schließlich ist anzunehmen, dass der Anteil an „Robin Hood OA“ in dieser Kategorie besonders hoch ist, da mit der Speicherung auf einigen der betreffenden Server keinerlei Kontrolle der Uploads verknüpft ist. Dazu zählen auch persönliche Homepages.

Archambault et al. verwenden Daten aus $\mathrm{Scopus}^{7}$, um aus allen dort verzeichneten Artikeln, die zwischen 1996 und 2013 erschienen sind, geographisch und zeitlich gut verteilte Zufallsstichproben zu ziehen. Auch wenn der Report keine genauen Angaben darüber enthält, gehe ich davon aus, dass die Zuordnung der Artikel zu den einzelnen Ländern per Institutionszugehörigkeit der Autorlnnen vorgenommen wurde und nicht etwa aufgrund des Verlagsorts.

Artikel ab dem Jahr 2008 erhalten in der Studie besondere Aufmerksamkeit. Die geographische Abdeckung erstreckt sich auf alle Länder des Europäischen Forschungsraums (ERA) sowie Brasilien, Kanada, Japan und die USA. Wenn also im Folgenden diese Länder unter dem Titel „Welt“ zusammengefasst werden, handelt es sich um eine grobe Vereinfachung. Wa- 
rum diese geographische Auswahl getroffen wurde, wird im Report nicht begründet.

Auch die verwendete Klassifikation der Disziplinen - 22 an der Zahl wird nicht näher erläutert. Es handelt sich jedenfalls weder um die Klassifikation von Scopus noch um jene von DOAJ. Leider ist sie m.E. nicht sehr eingängig, weshalb ich mich dazu entschlossen habe, die englischen Bezeichnungen beizubehalten, um durch eine Übersetzung das Ergebnis nicht zu verfälschen.

Bei der Datenerhebung wurde zu unterschiedlichen Zeitpunkten die Zugänglichkeit der Artikel - gleich, zu welcher Version - getestet. Die hier weiterverwendeten Daten geben den Stand von April 2014 wieder.

Der Report bietet im Appendix Tabellen, die für jedes untersuchte Land und den Gesamtraum die jeweiligen Artikelstichproben für den Zeitraum 2008-2013 aufteilen, und zwar nach Disziplinen und OA-Typ. Ich habe mich auf den Vergleich der österreichischen Daten mit denen des insgesamt untersuchten Raums konzentriert. Dazu habe ich die entsprechenden Zahlenreihen mittels des Tools Tabula ${ }^{8}$ extrahiert. ${ }^{9}$ Diese Reihen enthielten für jeden Wert Korrekturfaktoren, die sich aufgrund der Methode von Science-Metrix ergaben. Mit diesen Angaben konnte ich jedoch keine Diagramme erstellen, weshalb ich aus der jeweils größten möglichen Abweichung im positiven und negativen Bereich den Mittelwert gebildet habe. Falls nicht anders angegeben, beziehe ich mich im Folgenden auf diese errechneten Mittelwerte, die aus für mich nicht nachvolziehbaren Gründen mitunter geringfügig von den im Report genannten Werten abweichen.

\section{Zentrale Ergebnisse der Studie von Science-Metrix}

Als zentrales Ergebnis des Reports gilt, dass im April 2014 mehr als die Hälfte der zwischen 2008 und 2013 erschienenen Artikel frei zugänglich waren. Das gilt für jedes der untersuchten Länder. Für einige Länder lässt sich sogar feststellen, dass über $70 \%$ der Artikel frei zugänglich waren, nämlich für Brasilien, die Niederlanden, Kroatien, Estland und Portugal. Gold OA ist in Osteuropa am meisten verbreitet, am wenigsten in Frankreich, Großbritannien und Belgien (25). Allein dieses Ergebnis weicht teilweise von meinen Erwartungen $a b$, da gerade Großbritannien für eine millionenschwere OA-Förderpolitik bekannt ist. ${ }^{10}$ Für Brasilien zeigt sich hier der Erfolg der frühen Investitionen in neue Open-Access-Journals und die Plattform SCIELO ${ }^{11}$. 
Die hohe OA-Rate Portugals ergibt sich vor allem aus dem verhältnismäßig hohen Anteil von Grünem OA (16\%). Zwar sind hier vergleichsweise starke Policies bzw. Mandate zum Open-Access-Zweitpublizieren in den institutionellen Repositorien in Kraft, aber in anderen Ländern ist dies noch viel eher der Fall: Laut dem Verzeichnis ROARMAP ${ }^{12}$ fordern zehn portugiesische Forschungseinrichtungen die Archivierung einer Version jeder Publikation ihrer Angehörigen in einem Repositorium. An drei dieser portugiesischen Einrichtungen wird den Autorlnnen darüber hinaus vorgeschrieben, dass diese Publikationen auch frei zugänglich zu machen sind. In allen durch Archambault et al. untersuchten Ländern gibt es nach ROARMAP vergleichbar starke Policies an 116 Einrichtungen. Mehr als drei Viertel dieser Einrichtungen befinden sich - jeweils zu etwa gleichen Teilen - in Großbritannien und in den USA, wo es sehr viel mehr Forschungseinrichtungen gibt als in Portugal - sowie in Finnland. Interessanterweise liegt Finnland bezüglich Grün-OA nur im Mittelfeld (9\%) und hat auch sonst OA-Raten, die z. B. jenen von Österreich ähnlich sind. Eine vergleichbare Verbreitung von OA-Mandaten wie in Portugal findet sich sonst noch in Schweden, Italien, Frankreich und Kanada.

Angesichts dieser Daten muss man zu dem Schluss kommen, dass OAMandate nicht automatisch zu einer Erhöhung des Anteils frei zugänglicher Publikationen führen. Leider können an dieser Stelle keine schlüssigen Erklärungen für die hohen OA-Raten von den Niederlanden, Kroatien, Estland und Portugal entwickelt werden.

Interessant ist, dass beträchtliche Anteile der Stichproben z. B. 2012 noch zugänglich waren, 2014 aber nicht mehr. Die Ursachen dafür könnten Promotion-Aktionen von Verlagen sein, die temporär die Artikel zugänglich machten, oder das schlichte Verschwinden des Contents - ein trotz aller Bemühungen um Langzeitarchivierung noch immer sehr gravierendes Problem (28).

Die Verteilung der OA-Raten in den einzelnen Disziplinen ist erwartungsgemäß sehr ungleich (siehe Abb. 1). Für Artikel der Jahre 2011-2013 lässt sich dies daran ablesen, dass in nur sieben von 22 Disziplinen mehr als $50 \%$ frei zugänglich waren. In General Science \& Technology liegt die OARate bei 70\% (mit Korrekturfaktor sogar bei 90\%; zur Methode siehe Archambault et al. 2014, S. 8). Um 60\% OA weisen Biomedical Research, Mathematics \& Statistics und Biology auf. Am anderen Ende des Spektrums bilden die Visual \& Performing Arts mit 22\% das Schlusslicht, gefolgt von den Communication \& Textual Studies (28\%) sowie den Historical Studies, Engineering und Philosophy \& Theology mit jeweils etwa 30\% OA. Überraschen mag, dass der Gold-OA-Anteil in den Social Sciences jenen von „Green OA“ 


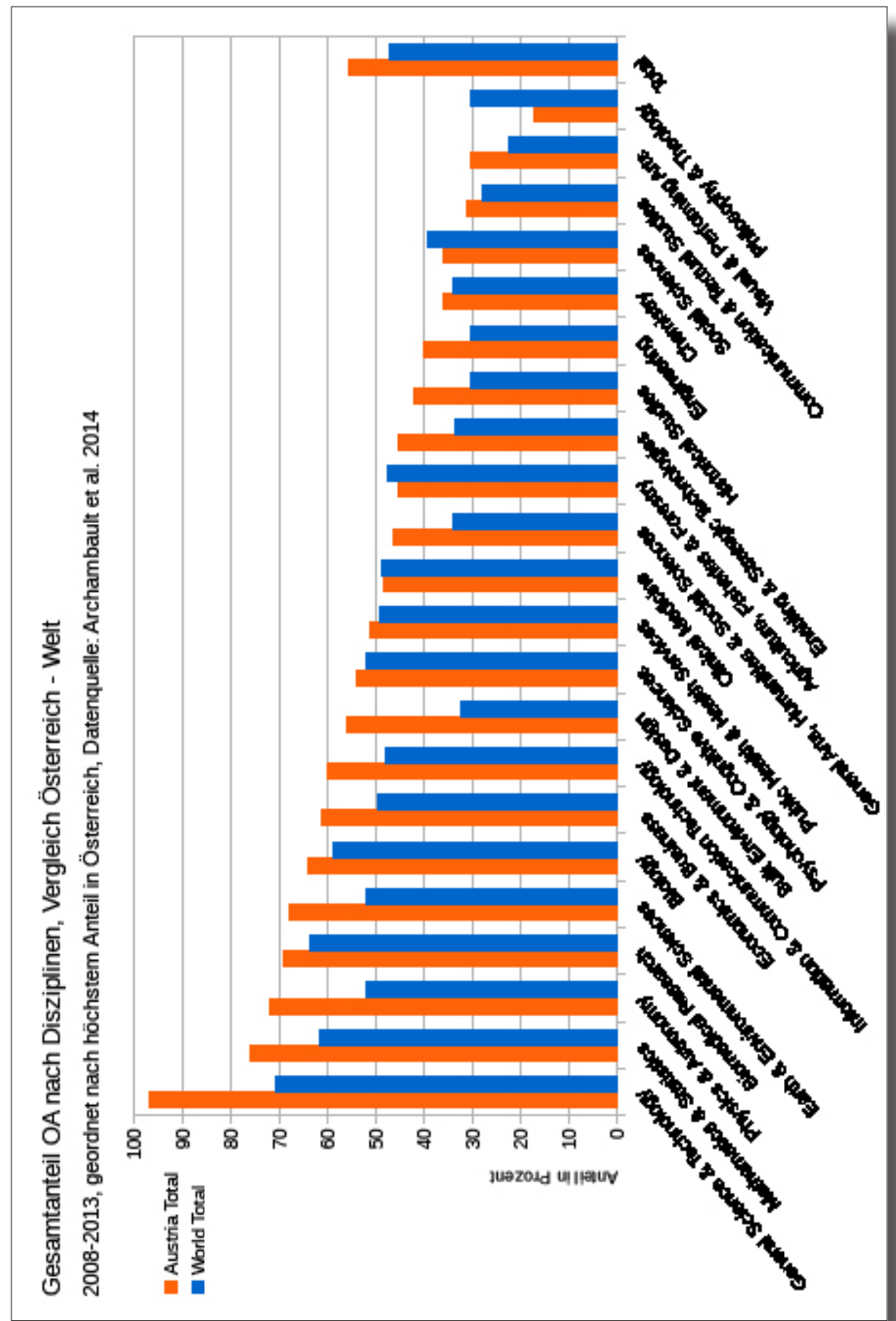

Abb. 1: Open-Access-Raten ohne Korrekturfaktor nach Disziplinen in Österreich und dem gesamten untersuchten Raum. Eigene Grafik nach Daten von Archambault et al. 2014. 
mit $8 \%$ gegenüber $6 \%$ sogar überragt. Disziplinübergreifend liegen die Sozialwissenschaften in Bezug auf Gold-OA nur noch knapp unter dem Durchschnitt und z. B. fast gleichauf mit Chemie.

Die höchste OA-Wachstumsrate 2008-2012 verzeichnet ebenfalls das Feld General Science \& Technology (19). Gefolgt von den Enabling \& Strategic Technologies und den Public Health and Health Services sowie der Clinical Medicine. In den Visual \& Performing Arts und im Built \& Environment Design sind die OA-Wachstumsraten trotz der geringen OA-Raten vergleichsweise hoch. Außerdem gibt es auch Disziplinen, in denen eine OASchrumpfung zu verzeichnen ist, vor allem in den General Arts, Humanities \& Social Sciences sowie in jenen Fachbereichen, denen eine gewisse (Gold-) OA-Resistenz attestiert wird, wie den Wirtschaftswissenschaften und der Psychologie.

Die Nennung dieser innerdisziplinär-relativen Wachstumsraten kann irreführend sein, da diese immer vor dem Hintergrund des generellen Wachstums des Artikeloutputs betrachtet werden müssen (in Scopus 6,6\% jährlicher Zuwachs; S. 10). Deshalb hat Science-Metrix auch noch einmal aus den absoluten Artikelanzahlen Wachstumsraten kalkuliert, die ein anderes Bild zeichnen (siehe Abb. 2): in keinem Fachbereich kam es zu einer Verringerung der Anzahl von OA-Artikeln, und in absoluten Zahlen ist das Wachstum in den Visual \& Performing Arts sowie im Built \& Environment Design sogar noch deutlicher. Auch die Social Sciences und die Communication \& Textual Studies fallen positiv auf, in begrenztem Maße sogar Philosophy \& Theology und die Historical Studies.

Für die Gesamtergebnisse spielen Embargos, also die erst nach sechs bis 36 Monaten durch den Verlag ermöglichte Zurverfügungstellung eines Artikels, eine recht große Rolle (10f.). Ist die OA-Rate für Artikel aus den Jahren 2009-2012 relativ stabil, bricht sie für jüngere Artikel stark ein.

Der Anteil von Grünem OA ist seit 2004 weltweit nicht mehr gewachsen und stagniert bei etwa 6\% (13). Auch die Restkategorie "Other OA“ wächst seit 2009 nicht mehr und macht stabil etwa $32 \%$ aus (15). In beiden Kategorien wurden sogar sinkende Anteile gemessen, was sich jedoch bei erneuter Messung in einigen Jahren wahrscheinlich ausgleichen wird. Die OA-Wachstumsrate seit 2009 ist also nahezu ausschließlich Gold OA zu verdanken, dessen Wachstumsrate zwischen 1996 und 2012 jährlich bei $24 \%$ lag, aktuell jedoch leicht sinkt, was auf Unregelmäßigkeiten in der Datenbasis zurückzuführen sein könnte (14).

Wenig überraschend ist, dass der Grüne Weg vor allem in Physics \& Astronomy relevant ist (25\%). Der fachspezifische Preprintserver arXiv.org ${ }^{13}$ dürfte auch der Grund sein, weshalb die Rate in Mathematics \& Statistics 


\begin{tabular}{|c|c|c|c|c|c|c|}
\hline \multirow{2}{*}{ Field } & \multirow{2}{*}{$\begin{array}{c}\text { Green } \\
\% \\
\end{array}$} & \multirow{2}{*}{$\begin{array}{c}\text { Gold } \\
\%\end{array}$} & \multicolumn{2}{|c|}{ Other Total } & \multicolumn{2}{|c|}{ OA Growth } \\
\hline & & & $\%$ & $\%$ & Trend & Index \\
\hline Agriculture, Fisheries \& Forestry & 2.8 & 16.1 & 46.9 & 53.8 & 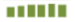 & 1.16 \\
\hline Biology & 3.7 & 17.0 & 57.7 & 66.2 & mandu & 1.12 \\
\hline Biomedical Research & 2.1 & 12.4 & 61.6 & 70.6 & andinI & 1.09 \\
\hline Built Environment \& Design & 4.6 & 3.5 & 33 & 37.5 & mantin & 1.33 \\
\hline Chemistry & 1.8 & 9.5 & 33.6 & 38.5 & annun & 1.18 \\
\hline Clinical Medicine & 2.2 & 14.8 & 49.1 & 56.3 & mandu & 1.19 \\
\hline Communication \& Textual Studies & 3.6 & 8.7 & 27 & 30.9 & mantiln & 1.27 \\
\hline Earth \& Environmental Sciences & 5.6 & 8.1 & 50.4 & 57.8 & anduid & 1.08 \\
\hline Economics \& Business & 11.3 & 5.4 & 47.9 & 54.9 & andula & 1.16 \\
\hline Enabling \& Strategic Technobgies & 2.7 & 9.3 & 34.3 & 39.3 & mantal & 1.34 \\
\hline Engineering & 3.2 & 4.1 & 30.2 & 34.6 & anturie & 1.20 \\
\hline Gen. Arts, Humanities \& Social Sciences* & 3.5 & 2.6 & 31.3 & 35.9 & allating & 1.04 \\
\hline Gen. Science \& Technology & 3.9 & 58.0 & 78.3 & 89.7 & $-\ldots=\mathbf{H}$ & 2.43 \\
\hline Historical Studies & 2.5 & 7.2 & 30 & 34.4 & mallum & 1.18 \\
\hline Information \& Communication Tech. & 8.7 & 12.4 & 47.1 & 54.0 & Eminn & 1.10 \\
\hline Mathematics \& Statistics & 24.3 & 11.4 & 58.9 & 67.6 & manill & 1.04 \\
\hline Philosophy \& Theology & 5.1 & 5.1 & 30 & 34.7 & manth & 1.24 \\
\hline Physics \& Astronomy & 25.6 & 5.1 & 51.8 & 59.4 & mantul| & 1.09 \\
\hline Psychology \& Cognitive Sciences & 3.6 & 5.6 & 50.4 & 57.7 & mann & 1.10 \\
\hline Public Health \& Health Services & 3.0 & 15.8 & 49.9 & 57.2 & mandu & 1.26 \\
\hline Social Sciences & 5.2 & 8.7 & 38.1 & 43.7 & analla & 1.28 \\
\hline Visual \& Performing Arts* & 2.9 & 2.8 & 23.3 & 26.7 & 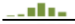 & 1.42 \\
\hline Total** & 5.9 & 12.1 & 46.9 & 53.7 & 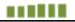 & 1.19 \\
\hline
\end{tabular}

Abb. 2: Open-Access-Raten 2011-2013 nach Disziplinen und OA-Typ für den gesamten untersuchten Raum inklusive einer Darstellung des jeweiligen Wachstumstrends, kalkuliert nach der absoluten Anzahl der verfügbaren Artikel 2011-2012, verglichen mit 2008-2009. Grafik von Archambault et al. 2014, S. 22, vereinfacht. Dort finden sich auch relative Wachstumszahlen.

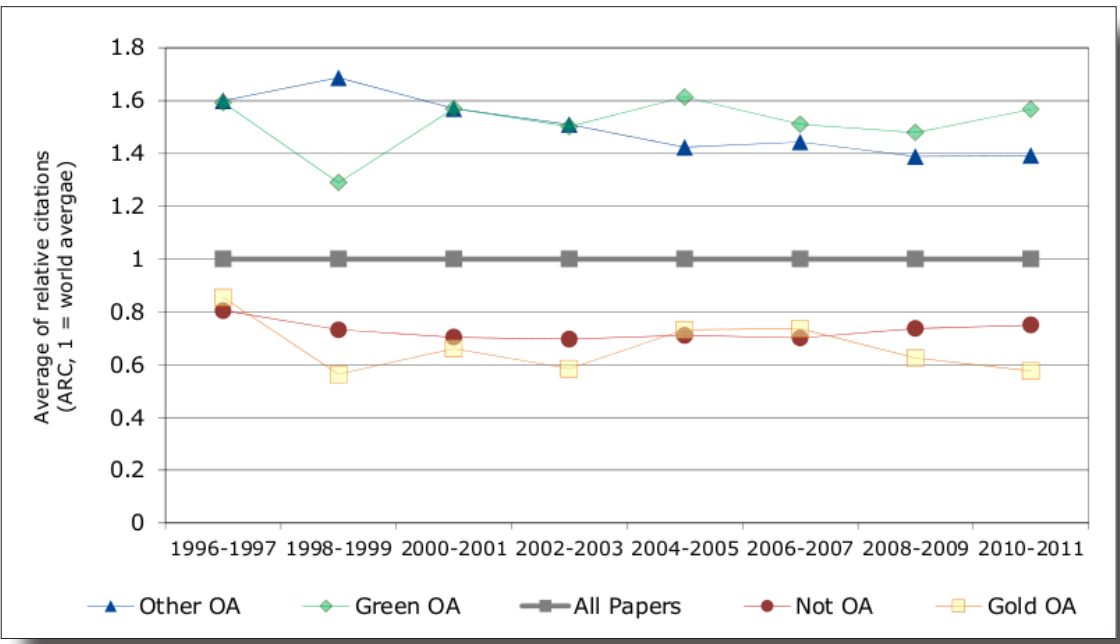

Abb. 3: Zitationsvor- und -nachteile bei den unterschiedlichen OA- bzw. Nicht-OA-Typen, 1996-2013. Grafik von Archambault et al. 2014, S.18. 
ähnlich hoch ist (23\%). Economics \& Business weisen mit $12 \%$ die höchste Grün-OA-Rate in den Geistes- und Sozialwissenschaften auf.

Schließlich untersucht Science-Metrix auch die Zitationsvorteile, die sich vermeintlich durch OA ergeben. Hierzu wurde jeweils das „Average of Relative Citation (ARC)“ berechnet, eine Kennzahl, die Unterschiede im Zitierverhalten unter den Disziplinen zu normalisieren versucht. Anhand von ca. 200.000 Artikeln ist abzulesen, dass die Wahrscheinlichkeit, zitiert zu werden, unter OA $40 \%$ höher liegt - hinter einer Pay- oder sonstigen Wall jedoch 27\% niedriger als der Durchschnitt. Dabei spielt auch eine Rolle, welcher OA-Typ gewählt wurde: Während Gold-OA in etwa gleichauf mit Nicht-OA einen Zitationsnachteil hat, liegt Grün-OA mit „Other OA“ weit im positiven Bereich (Abb. 3).

Interessant ist, dass in einigen Fachbereichen die Wahrscheinlichkeit, zitiert zu werden, wenn die Artikel in einem Repositorium archiviert und zugänglich gemacht wurden, sehr viel höher ist als in anderen: dazu gehören die General Science \& Technology, Historical Studies und Visual \& Performing Arts. Bemerkenswert ist auch, dass in den meisten Feldern, in denen das Publizieren in Repositorien einen guten Zitationsvorteil verschafft, dies auch für die Verwendung des fragwürdigen Typs „Other OA“ gilt. Schließlich sollen jene Disziplinen hervorgehoben werden, in denen Gold-OAJournals bereits ausreichend etabliert sind, um gegenüber Nicht-OA einen deutlichen Zitationsvorteil zu verschaffen: wenig überraschend zählen dazu die General Science \& Technology, Physics \& Astronomy, Biomedical Research und die Earth \& Environmental Sciences. Erkennbar ist jedoch bereits der sich abzeichnende Zitationsvorteil in Philosophy \& Theology sowie in Information \& Communication Technology.

\section{Hervorzuhebende Ergebnisse für Österreich}

Zunächst fällt auf, dass die Daten aus Österreich gegenüber jenen aus dem untersuchten Gesamtraum in nur wenigen Disziplinen deutlich abweichen. Lässt man die Unterschiede in den Anteilen der OA-Typen beiseite (s. Abb. 4a und 4b), muss Österreich nach diesen Daten als Kernland des OA gelten: In fast allen Disziplinen liegt der OA-Anteil in Österreich höher als international (Abb. 1). Ausnahmen bilden folgende Disziplinen: Philosophy \& Theology mit deutlichem, die Social Sciences, Agriculture, Fisheries \& Forestry sowie Clinical Medicine mit geringem Abstand.

Für Philosophy \& Theology lässt sich davon ein Handlungsbedarf für österreichische OA-Beauftragte ablesen, zumal die aus diesem Fach zugäng- 
lichen Artikel dem Other-OA-Typus zugeordnet wurden. Den Erhebungen zufolge wird weder der Grüne noch der Goldene Weg von den österreichischen PhilosophInnen und TheologInnen beschritten. International ist das Feld bei beiden Typen keineswegs das Schlusslicht, was es unwahrscheinlich macht, dass die unzureichende Abdeckung von Scopus die Ursache dieses Ergebnisses für Österreich ist. Als Hauptgrund dafür lässt sich vielmehr annehmen, dass hier hauptsächlich deutschsprachig publiziert wird und das Directory of Open Access Journals (DOAJ) ${ }^{14}$ im ganzen Feld nur neun Journals aufweist, die hauptsächlich Beiträge in deutscher Sprache veröffentlichen, übrigens primär in den Religionswissenschaften. ${ }^{15}$ Es ist kein einziges Journal darunter, dass sich originären Themen der Philosophie widmet. Die neun Zeitschriften sind fast alle sehr jung: Blendet man die eine Ausnahme, ein Journal, das bereits 1998 startete, aus, gibt es sie durchschnittlich erst seit 2006.

Es wäre interessant zu erfahren, in welchen Journals die international $5 \%$ Gold-OA-Artikel in Philosophy \& Theology publiziert wurden. Auch würde ein detaillierter Einblick in die Österreich-Stichprobe wahrscheinlich aufschlussreich sein, denn Scopus listet für den betreffenden Zeitraum im Fachbereich Arts \& Humanties gerade einmal 1.484 Artikel aus Österreich - so viel wie ein Sechstel der Gesamtstichprobe für Österreich. Was den Grünen Weg angeht, so muss angemerkt werden, dass in Österreich - neben den britischen PhilPapers ${ }^{16}$ - ein offenes Fachrepositorium für diese Disziplin betrieben wird: Im Sammelpunkt ${ }^{17}$ des Instituts für Philosophie der Universität Wien sind aus dem betreffenden Zeitraum allerdings nur 61 Artikel zugänglich. ${ }^{18}$

Einige Disziplinen fallen aufgrund ihres überragenden österreichischen Ergebnisses auf: Die Earth \& Environmental Sciences überholen in Österreich bei allen drei OA-Typen die internationalen Raten; vor allem ist der Anteil von $17 \%$ Gold-OA gegenüber den internationalen $8 \%$ hervorzuheben. Das gleiche gilt gesteigert auch für General Science \& Technology, wo der OA-Anteil in Österreich bald $100 \%$ betragen wird, während international nicht einmal drei Viertel erreicht werden. Dieses Feld ist gleichzeitig das einzige, in dem der Gold-OA-Anteil dafür die größte Rolle spielt. Außerdem sind die beiden genannten Felder die einzigen, bei denen der Gold-OA-Anteil höher ist als im internationalen Schnitt. Als Grund für die im Hinblick auf Gold-OA unterdurchschnittlichen Ergebnisse der anderen Disziplinen kann vermutet werden, dass es im untersuchten Zeitraum in Österreich abgesehen von FWF-Projekten kaum Förderungen für das OA-Publizieren gab. Mit der sukzessiven Einrichtung von Publikationsfonds an den Universitäten und anderen Forschungseinrichtungen ändert sich das derzeit. ${ }^{19}$ 


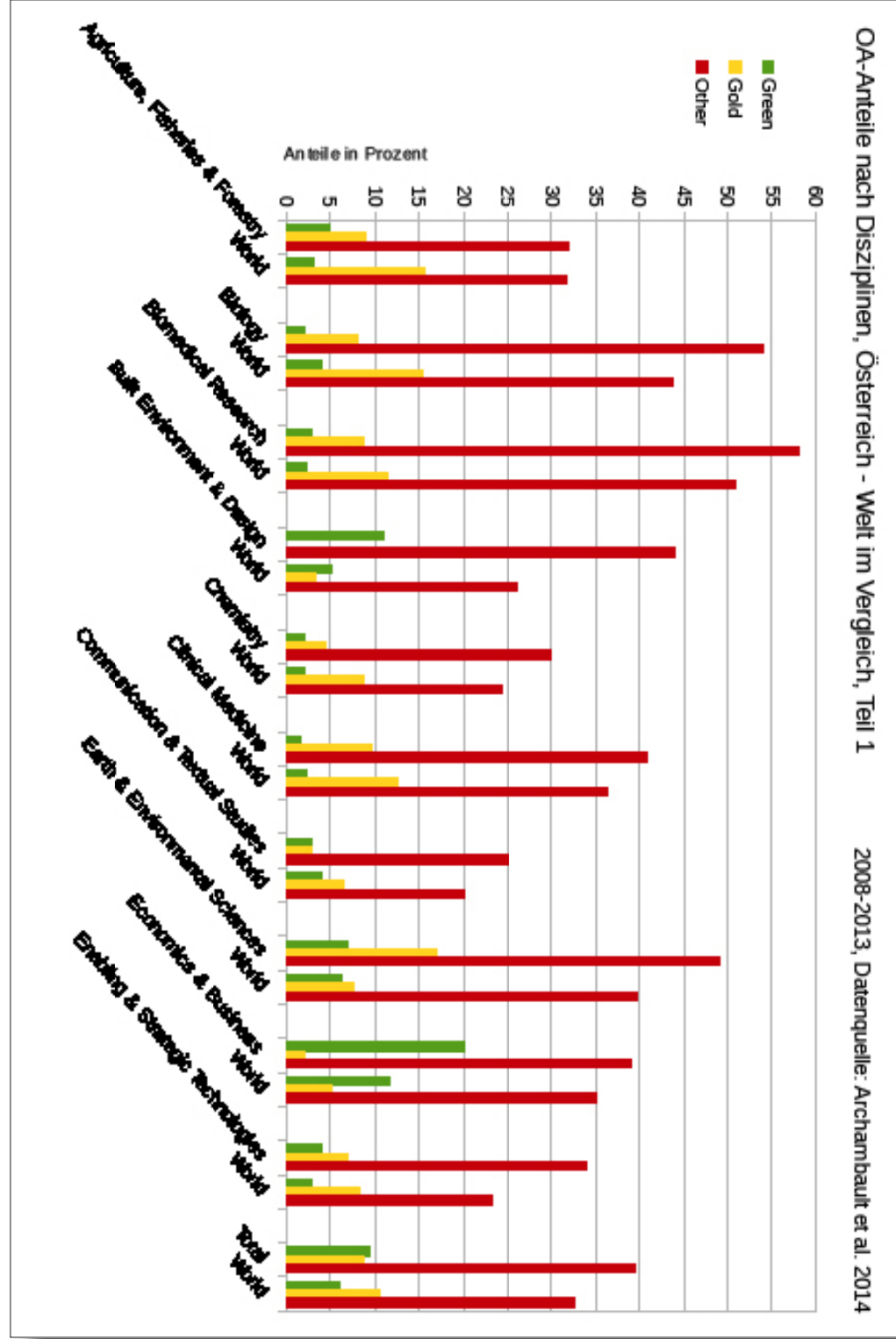

Abb. 4a: Anteile von OA-Publikationen in den unterschiedlichen Disziplinen, alphabetisch geordnet, nach OA-Typen, Teil 1. Das erste Säulenbündel je Disziplinen zeigt die Ergebnisse für Österreich, das zweite jene für den untersuchten Gesamtraum. Eigene Grafik nach Daten von Archambault et al. 2014. 


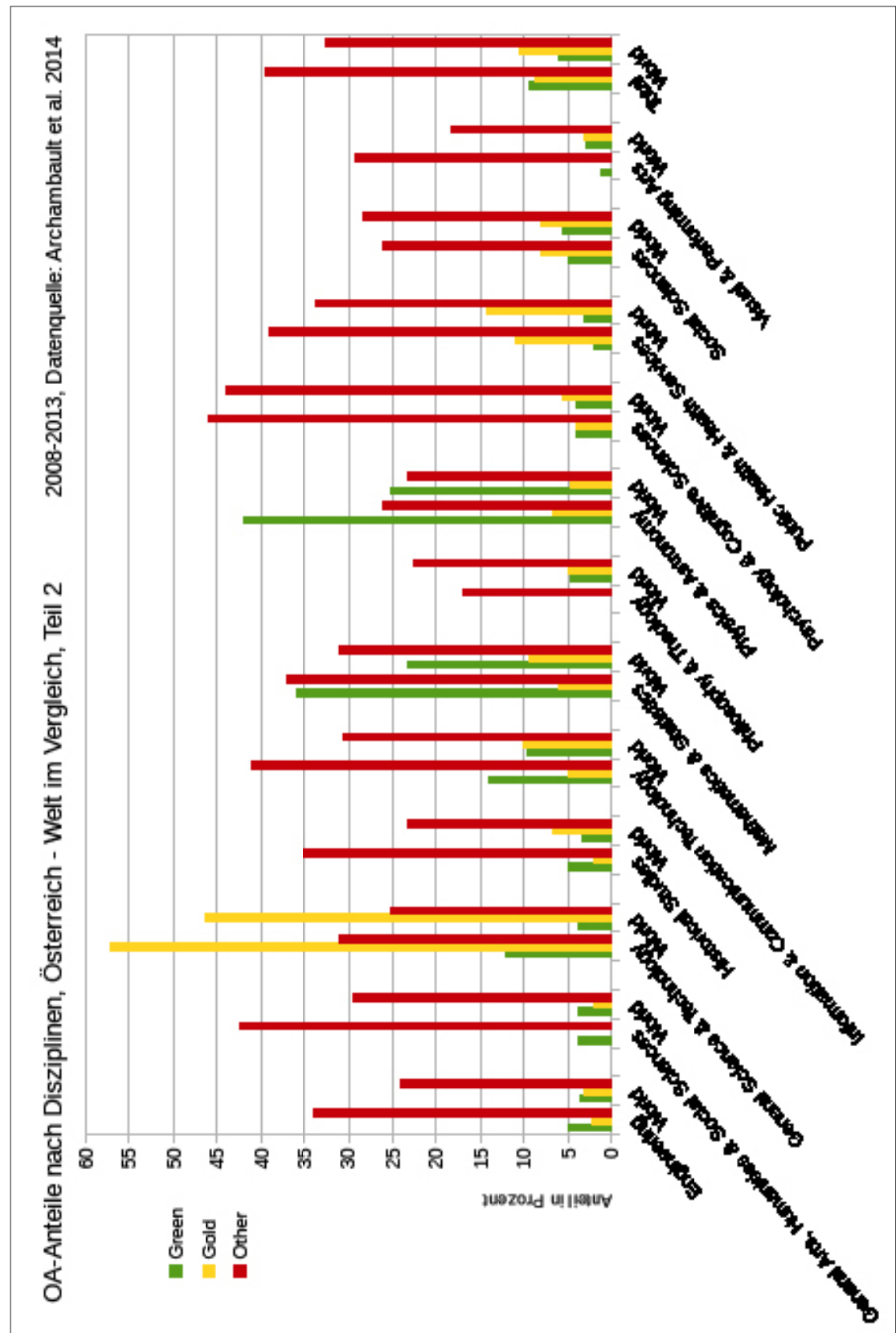

Abb. 4b: Beschreibung siehe Abbildung 4a, Teil 2. 
„Green $O A$ “ ist in Österreich mit einem disziplinübergreifend etwa 9\%igen Anteil erfolgreicher als international (6\%). Diese Tendenz betrifft jedoch nur einige Disziplinen, die mit überdurchschnittlichen Anteilen hervorragen. Andere verschwinden in dieser Hinsicht nahezu. Die vergleichsweise späte Einrichtung von institutionellen Repositorien, die mit wenigen Ausnahmen erst aufgrund des entsprechenden Serviceangebots der Österreichischen Bibliotheksverbund und Service GmbH (OBVSG) ${ }^{20}$ seit März 2013 stattfindet, könnte dieser Tendenz zukünftig einen weiteren Schub geben. Den größten Anteil am Erfolg des Grünen Wegs in Österreich hat jedoch eindeutig arXiv.org, denn in Physics \& Astronomy (42\%) sowie in Mathematics \& Statistics (36\%) führt der hohe Grün-OA-Anteil sogar dazu, dass Österreich bei der Gesamt-OA-Rate in diesen Feldern um 7-10\% vor den internationalen Ergebnissen liegt.

Zu den weiteren Disziplinen, in denen der Grüne Weg in Österreich außerordentlich erfolgreich bzw. noch erfolgreicher als international ist, gehört z. B. auch Economics \& Business, wo Österreich mit $20 \%$ Grün-OA deutlich über dem internationalen Schnitt von $12 \%$ liegt, sowie General Science \& Technology (12\% gegenüber 4\% international). Überraschender sind die Ergebnisse für Information \& Communication Technology und Built \& Environment Design: Beide Fachbereiche haben keine ausgesprochene Preprint-Kultur und befinden sich bei der allgemeinen OA-Adaption im Mittelfeld. Die mangelnde österreichische Adaption von Gold-OA in diesen Fachbereichen kann durch Grün-OA und „Other OA“ überkompensiert werden.

Nicht zu übersehen ist, dass der Anteil von „Other OA“ in fast allen Disziplinen in Österreich höher ist als international. ${ }^{21}$ Während davon ausgegangen werden kann, dass sich für die Naturwissenschaften hierunter zahlreiche Hybrid-OA-Artikel finden dürften, die zumeist Creative-Commons-Lizenzen aufweisen, ist eine hohe Verbreitung des Freikaufens von Artikeln in den Sozial- und Geisteswissenschaften kaum anzunehmen. Die hohen Raten könnten darauf hindeuten, dass sich die Förderpolitik des Wissenschaftsfonds (FWF), der Hybridpublikationen für geförderte Projekte finanziert, niederschlägt. Desweiteren wäre es interessant, genauer zu eruieren, wie hoch der Anteil von einst unter einer Embargo-Frist publizierten, aber dann durch die Zeitschrift verzögert zugänglich gemachten Artikeln ist. Die Daten könnten auch darauf verweisen, dass die Motivation, die eigenen Forschungsgebnisse zugänglich zu machen, vorhanden ist, es jedoch an Know-How in rechtlichen Fragen und an einem Bewusstsein für die Notwendigkeit von persistenter Referenzierbarkeit fehlt. Leider lässt sich anhand der vorliegenden Daten keine genauere Aussage treffen. 
Besonders hohe Anteile von „Other OA“ finden sich - den internationalen Daten in wenigem nachstehend - in Psychology \& Cognitive Sciences (46\%, bei je 4\% Gold und Grün, möglicherweise mit einem Anteil Hybrid-OA) und in General Arts, Humanities \& Social Sciences, wo $42 \%$ in Österreich international $30 \%$ gegenüberstehen. Dieser hohe Anteil bedingt auch, im Hinblick auf den Gesamt-OA-Anteil, die Positionierung des Fachbereichs im unteren Mittelfeld, obwohl es international den einen oder anderen Platz tiefer gerankt ist. In den Visual \& Performing Arts wird nahezu die GesamtOA-Rate von gut $30 \%$ für Österreich in diesem Feld aus dem Typ „Other OA" gespeist, was international durchaus nicht der Fall ist.

Es zeigt sich - wenig überraschend -, dass sowohl die niedrigsten OARaten als auch die starke Frequentierung des „anderen“ Weges für die Geistes- und teilweise auch für die Sozialwissenschaften charakteristisch sind. Für die Suche nach den Ursachen kann die Study of Open Access Publishing (SOAP; Dallmeier-Tiessen, Darby et al. 2011) zur Rate gezogen werden. Die Daten dieser Studie sind ebenfalls frei zugänglich ${ }^{22}$ und wurden für Österreich bereits von Bruno Bauer (2011) und mir (Schmidt 2014) ausgewertet. Leider haben sich nur wenige Geistes- und SozialwissenschaftlerInnen an der Studie beteiligt, nämlich 138 (fachübergreifend 462) ForscherInnen aus Österreich.

Wie Abb. 5 zeigt, geben diese zumeist an, dass sie OA gegenüber grundsätzlich positiv eingestellt sind. Auch finden die Forscherlnnen nicht, dass es an Open-Access-Journals im eigenen Feld mangelt. Schaue ich mir jedoch die Faktoren an, welche die Wahl eines Journals bestimmen, wird deutlich, warum sich die 102 Geistes- und Sozialwissenschaftlerlnnen, die hier Gründe angegeben haben, letztendlich doch für Subskriptionsjournals entscheiden (Abb. 6): Nach wie vor ist Open Access - wenn es auch bekanntermaßen mit einem Zitationsvorteil einhergeht (Abb. 3) - nichts, was gegenüber der höheren Reputation von Subskriptionsjournals überzeugen würde. Die Relevanz für die Community und das Prestige des Journals sind ausschlaggebend (Abb. 6). Immerhin ist die Copyright Policy für fast die Hälfte der Befragten ein wichtiger oder sehr wichtiger Faktor. Die beiden Faktoren „Copyright Policy“ und „Open Access“ sind jedoch mit Abstand die unwichtigsten Faktoren. Übrigens sind dabei kaum disziplinäre Besonderheiten erkennbar.

\section{Fazit}

Auch wenn die Methode der Datenerhebung von Science-Metrix nicht immer nachvollziehbar erscheint, sind die gewonnenen Daten und Ergebnisse 


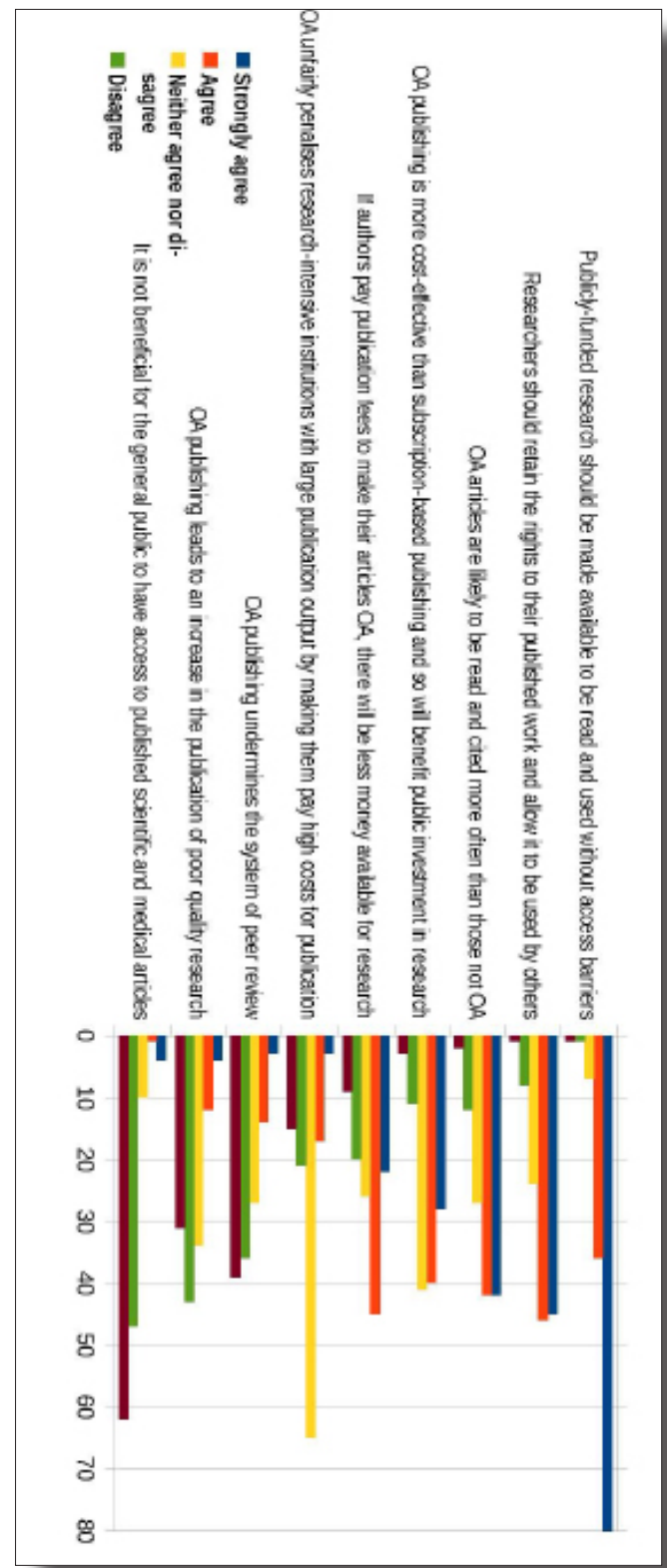

Abb. 5: Meinungen zu OA von Österreichischen Geistes- und Sozialwissenschaftlerlnnen, geordnet nach „Strongly agree + Agree“. N=125. Eigene Grafik nach Daten von DallmeierTiessen, Darby et al. 2011, siehe Fn. 22. 


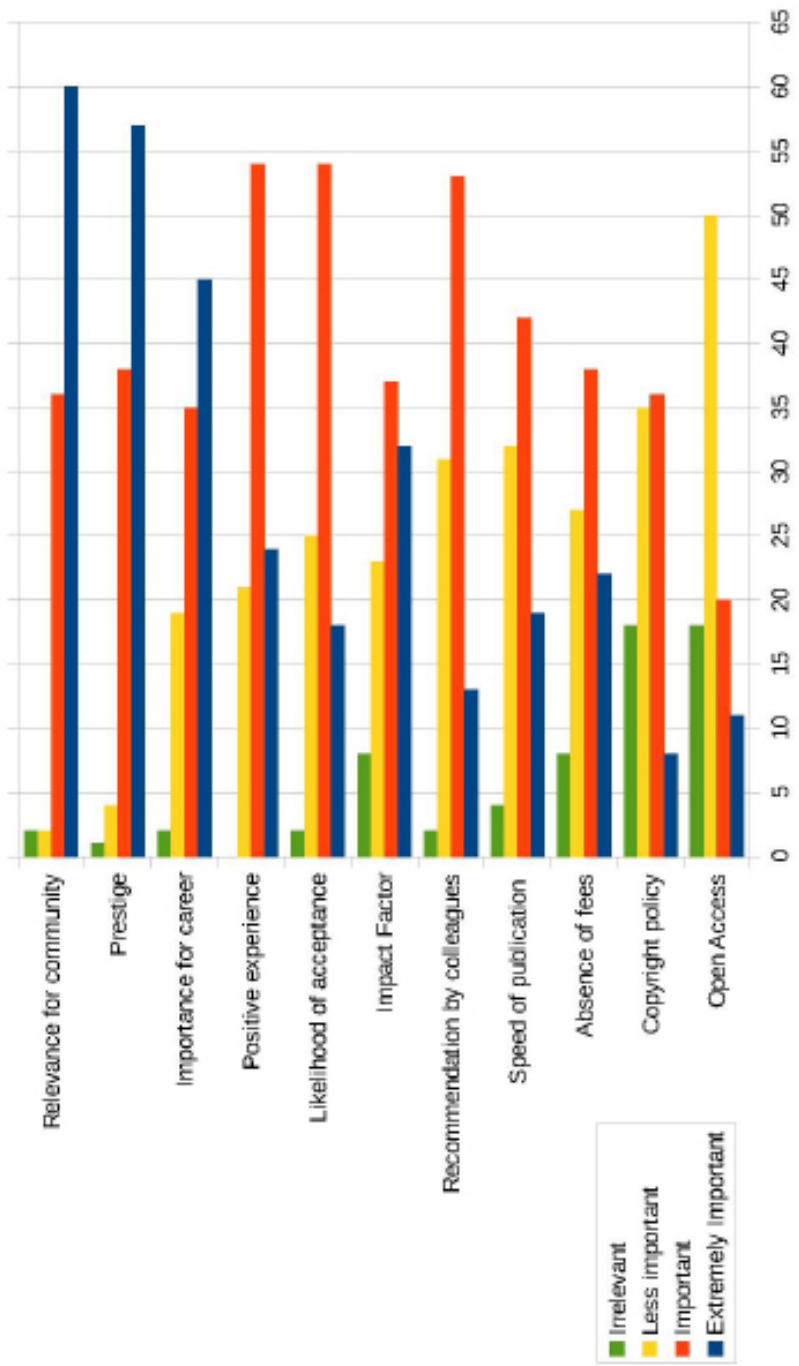

Abb. 6: Faktoren bei der Wahl eines Journals durch Österreichische Geistes- und Sozialwissenschaftlerlnnen, geordnet nach „Extremely important + Important“. N=102. Eigene Grafik nach Daten von Dallmeier-Tiessen, Darby et al. 2011, siehe Fn. 22. 
eine wertvolle Grundlage für die Beurteilung der Entwicklung des internationalen Publikationswesens. Für die Österreichische OA-Community sind die Ergebnisse dieser Datenauswertung nicht nur größtenteils überraschend erfreulich, sondern auch hilfreich zur Unterstützung der Diskussion um eine österreichweite OA-Strategie ${ }^{23}$ einerseits und für die Entwicklung von Initiativen an den jeweiligen Institutionen andererseits, denn für die OA-Community besteht deutlicher Handlungsbedarf: Zum Einen sollten die Gründe für die niedrigen OA-Raten in Philosophy \& Theology untersucht werden, um die Forschungsgebnisse dieses Fachbereichs besser zugänglich zu machen. Zum Anderen ist das Publizieren in Open-Access-Zeitschriften in Österreich unterdurchschnittllich verbreitet. Ausgenommen davon sind die Fachbereiche Earth \& Environmental Sciences sowie General Science \& Technology.

Obwohl an vielen österreichischen Institutionen gerade erst Repositorien eingeführt wurden, ist der Grüne Weg in Österreich bereits durchschnittlich besser etabliert als im untersuchten Gesamtraum. Den größten Anteil an dieser Verbreitung haben allerdings jene Disziplinen, die mit arXiv.org über einen hervorragend funktionierendes Fachrepositorium und eine Preprint-Kultur verfügen. Teile der Sozialwissenschaften schließen sich dem mit eigenen Fachrepositorien an.

Die Suche nach Erklärungen für die hohen OA-Raten einiger Länder hat gezeigt, dass die Einführung von institutionellen Verpflichtungen zum OAPublizieren, von OA-Mandaten, keine Maßnahme mit Erfolgsgarantie ist. Hierzu sollte jedoch intensiver geforscht werden, bevor diese Maßnahme als Möglichkeit ausgeschlossen wird.

Die außerordentlich starke Verbreitung des „Anderen“ Typus in Österreich lässt - mit allen Unsicherheiten, die eine Restkategorie mit sich bringt - darauf schließen, dass die Themen Lizenzierung und Langzeitarchivierung sowie persistente Referenzierbarkeit mitunter noch Neuland sind. Eine Verankerung dieser Themen in Curricula könnte hier Abhilfe schaffen. Die Bekanntheit von empfehlenswerten Open-Access-Journals zu fördern, damit diese schneller Reputation aufbauen können, würde den Goldenen Weg des Open Access stärken. Außerdem sollte die Open-Access-Öffenlichkeitsarbeit ausgeweitet werden, bestenfalls österreichweit koordiniert über das Open Access Network Austria (OANA), um die Vorteile des Grünen gegenüber dem „Anderen“Weg bekannter zu machen. 
Mag. ${ }^{a}$ Nora Schmidt Universität Wien, DLE Bibliotheks- und Archivwesen

Open Access Office E-Mail: nora.schmidt@univie.ac.at

\section{Literatur}

Archambault, É.; Amyot, D.; Deschamps, P.; Nicol, A.; Provencher, F.; Rebout, L. \& Roberge, G. (2014): Proportion of Open Access Papers Published in Peer-Reviewed Journals at the European and World Levels - 1996-2013. RTD-B6-PP-2011-2: Study to develop a set of indicators to measure open access. European Commission, http://science-metrix. com/files/science-metrix/publications/d_1.8 sm_ec dg-rtd_proportion oa 1996-2013 v11p.pdf, zuletzt besucht am 21.05.2015.

Bauer, B. (2011): Österreichische Wissenschaftlerinnen und Wissenschaftler und der Goldene Weg zu Open Access: Ergebnisse aus der Study of Open Access Publishing (SOAP). Mitteilungen der VÖB 64, 413-428, http://hdl.handle.net/10760/16584.

Dallmeier-Tiessen, S.; Darby, R.; Goerner, B.; Hyppoelae, J.; Igo-Kemenes, P.; Kahn, D.; Lambert, S.; Lengenfelder, A.; Leonard, C.; Mele, S.; Nowicka, M.; Polydoratou, P.; Ross, D.; Ruiz-Perez, S.; Schimmer, R.; Swaisland, M. \& van der Stelt, W. (2011): Highlights from the SOAP project survey. What Scientists Think about Open Access Publishing, http://arxiv.org/abs/1101.5260, zuletzt besucht am 21.05.2015.

de Moya-Anegón, F.; Chinchilla-Rodríguez, Z.; Vargas-Quesada, B.; Corera-Álvarez, E.; Muñoz-Fernández, F. J.: González-Molina, A. \& Herrero-Solana, V. (2007): Coverage analysis of Scopus: A journal metric approach. Scientometrics 73(1), 53-78, doi:10.1007/s11192-007-1681-4.

Reding, S.; Gumpenberger, C.; Ovalle-Perandones, M.-A. \& Gorraiz, J. (2013): Über den Impakt von Gold Open Access Journals - Eine Analyse auf Makro-, Meso- und Mikroebene. Bibliometrie - Praxis und Forschung 2, http://nbn-resolving.de/urn:nbn:de:bvb:355-bpf-168-0.

Schmidt, N. (2014): Der Goldene Weg des Open Access zum funktionalen Publikationswesen. Handlungsoptionen für die Universität Wien, http://hdl.handle.net/10760/22655. 
1 Reding, Gumpenberger et al. haben 2013 die Anzahl österreichischer Publikationen in OA-Journals erhoben: 4\% aller österreichischen Publikationen der Jahre 2007-2011 finden sich in Journals, die sowohl in den Journal Citation Reports (JCR) als auch im Directory of Open Access Journals (DOAJ) verzeichnet sind.

2 Zahlen in Klammern verweisen aufSeitenangaben in dieser Publikation. Auf der Website von Science-Metrix finden sich außer dem hier referierten weitere Reports, die aus demselben Auftrag hervorgegangen sind: "State-of-art analysis of OA strategies to peer-review publications", "State-of-art analysis of OA strategies to scientific data", „Comparative analysis of the strengths and weaknesses of existing open access strategies, "Evolution of Open Access Policies and Availability, 1996-2013“, siehe http://science-metrix.com/en/publications/reports, zuletzt besucht am 21.05.2015.

3 Berlin Declaration on Open Access to Knowledge in the Sciences and Humanities, 22. Oktober 2003, http://openaccess.mpg.de/BerlinerErklaerung, zuletzt besucht am 21.05.2015.

4 The Directory of Open Access Repositories - OpenDOAR, http://opendoar.org/, zuletzt besucht am 21.05.2015.

5 Registry of Open Access Repositories, http://roar.eprints.org/, zuletzt besucht am 21.05.2015.

6 PubMed Central, http://www.ncbi.nlm.nih.gov/pmc/, zuletzt besucht am 21.05.2015.

7 Scopus, http://www.scopus.com/, zuletzt besucht am 21.05.2015. Zu beachten ist, dass die Datenbank sprachlich, geographisch und fachlich unausgewogen ist, siehe z. B. de Moya-Anegón et al. 2007. Dies wird von Archambault at al. nicht reflektiert.

8 An dieser Stelle möchte ich den Entwicklerlnnen der Open-SourceSoftware danken, die unter folgender Adresse downloadbar ist: http:// tabula.technology/, zuletzt besucht am 21.05.2015.

9 Die extrahierten und ausgewerteten Daten finden sich unter Schmidt, N. (2015): Proportion of Open Access Papers Published in Peer-Reviewed Journals in Austria 2008-2013, doi:10.5281/zenodo.17821.

$10 £ 10$ Mio. wurden den regierungsnahen britischen Förderorganisationen, den Research Councils, 2012 überreicht, um das Open-AccessPublizieren zu fördern, siehe die Pressemitteilung der Regierung, Government invests $£ 10$ million to help universities move to open access, 07.09.2012, https://www.gov.uk/government/news/government-invests-10-million-to-help-universities-move-to-open-access, zuletzt besucht am 21.05.2015. 
11 SciELO. Scientific Electronic Library Online, http://www.scielo.org, zuletzt besucht am 21.05.2015.

12 ROARMAP, http://roarmap.eprints.org/, zuletzt besucht am 21.05.2015. Der den folgenden Schlussfolgerungen zugrunde liegende Datensatz findet sich unter Schmidt, N. (2015): Policies of Research Institutions Requiring Open Access Depositing, doi:10.5281/zenodo.17822.

13 arXiv.org, http://arxiv.org/, zuletzt besucht am 21.05.2015.

14 Directory of Open Access Journals, http://doaj.org, zuletzt besucht am 21.05.2015.

15 Eruiert wurden diese Journals anhand der Erstnennung der deutschen Sprache. Die Titel dieser Zeitschriften lauten wie folgt: Aemaet; E-Journal Philosophie der Psychologie; Internationale Zeitschrift für Philosophie und Psychosomatik; Lectio Difficilior; Review of Biblical Literature; Theologie.Geschichte : Zeitschrift für Theologie und Kulturgeschichte; Journal für Philosophie und Psychiatrie; Theo-Web : Zeitschrift für Religionspädagogik; Zeitschrift für Junge Religionswissenschaft.

16 PhilPapers, http://philpapers.org, zuletzt besucht am 21.05.2015.

17 Sammelpunkt. Elektronisch archivierte Theorie, http://sammelpunkt. philo.at, zuletzt besucht am 21.05.2015.

18 Die Abfrage am 21.05.2015 lautete folgendermaßen: „Date is between 2008 and 2013 inclusive AND Item Type matches any of „Article“."

19 Leider gibt es derzeit noch keine Übersicht über alle österreichischen Publikationsfonds. Die erste Anlaufstelle wäre das Open Access Network Austria (OANA, http://www.oana.at). Folgende Fonds können genannt werden: Universität Wien (siehe http://openaccess.univie.ac.at/ foerderungen), Universität Salzburg (siehe http://www.uni-salzburg. at/index.php?id=67089), Technische Universität Wien (siehe http:// www.ub.tuwien.ac.at/openaccess), Medizinische Universität Graz (siehe http://www.medunigraz.at/open-access/faq/oa-foerderung) und Akademie der bildenden Künste Wien (der „Richtlinie des Rektorats zur Open-Access-Strategie“ zu entnehmen, siehe https://www.akbild. ac.at/Portal/kunst-forschung/open-access/akademie open $\% 20$ access\%20richtlinie.pdf), alle Webseiten zuletzt besucht am 21.05.2015.

20 OBVSG, Visual Library: Das Konsortialmodell der OBVSG, siehe https:// www.obvsg.at/services/visual-library, zuletzt besucht am 21.05.2015.

21 Dadurch, dass PubMed Central zu „Other OA“ beiträgt, aber dem Prinzip nach näher an „Green OA“ als an den sonstigen Plattformen gebaut ist, die "Other OA“ anbieten, können die Ergebnisse für Biomedical Research und Biology in dieser Hinsicht nicht beurteilt werden. 
22 Study of Open Access Publishing Data, http://bit.ly/ejuvKO, zuletzt besucht am 21.05.2015, Lizenz: CC 0, https://creativecommons.org/ publicdomain/zero/1.0/. Die extrahierten und ausgewerteten Daten finden sich unter Schmidt, N. (2015), Journal Selection Factors and Opinions about Open Access of Austrian Researchers in the Humanities and Social Sciences, doi:10.5281/zenodo.17824.

23 Im Rahmen des Open Access Network Austria (OANA) hat 2015 eine Arbeitsgruppe „Nationale Open Access Strategie“ ihre Arbeit aufgenommen, siehe http://www.oana.at/arbeitsgruppen-2015-2016/nationale-open-access-strategie, zuletzt besucht am 21.05.2015.

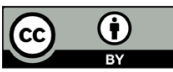

Dieses Werk ist lizenziert unter einer Creative-Commons-Lizenz Namensnennung 4.0 International 